\title{
TGF- $\beta 1$ and TIMP1 double directional rAAV targeted by UTMD in atherosclerotic vulnerable plaque
}

\author{
YIJIN SU*, CHANGSONG XU*, KUNYU LI, BO WANG, JUFANG CHEN, LONG LIU, \\ LIZHOU LIN, QINGQING DONG and LIANFANG DU \\ Department of Ultrasound, Shanghai General Hospital Affiliated to Nanjing Medical University, \\ Shanghai 200080, P.R. China
}

Received March 23, 2016; Accepted January 3, 2017

DOI: $10.3892 / \mathrm{etm} .2017 .4101$

\begin{abstract}
In the present study, we determined whether ultrasound-targeted microbubble destruction (UTMD) combined with dual targeting of transforming growth factor (TGF)- $\beta 1$ and tissue inhibitors of metalloproteinase (TIMP) 1 recombinant adeno-associated virus (rAAV) can stabilize atherosclerotic vulnerable plaques. First, we used rabbit model to detect the TGF- $\beta 1 /$ TIMP1 virus therapy result. H\&E staining was used to evaluate the tissues. The protein levels of TGF- $\beta 1$ and TIMP1 were detected in rabbit models. The THP-1 cells were induced into macrophages, and transfected with TGF- $\beta 1$ and TIMP1 rAAV under optimized UTMD. The expression of TGF- $\beta 1$ and TIMP1 was measured by RT-PCR and western blotting. We found that the apoptotic rates were induced when compared to the control group. The rAAV virus group showed a significant decrease in the p-ERT and p-AKT expression. These data support the hypothesis that TGF- $\beta 1$ and TIMP1 are crucial in the regulation of atherosclerotic plaques.
\end{abstract}

\section{Introduction}

Atherosclerosis (As) is a chronic progressive endothelial injury- and inflammation-caused vascular intimal lesion. The plaque ruptures after shedding embolus and subsequent thrombosis is the main cause of acute ischemic stroke of heart head blood-vessel, and plaque rupture almost occur on the basis of vulnerable plaques $(1,2)$. Therefore, vulnerable plaque stabilization is the precondition of prevention and treatment of atherosclerosis. Vulnerable plaque refers to those which are

Correspondence to: Dr Lianfang Du, Department of Ultrasound, Shanghai General Hospital Affiliated to Nanjing Medical University, 100 Haining Road, Shanghai 200080, P.R. China

E-mail: lianfang_du1@163.com

*Contributed equally

Key words: ultrasound-targeted microbubble destruction, atherosclerotic, transforming growth factor- $\beta 1$, tissue inhibitors of metalloproteinase 1 , apoptosis unstable and thrombophilia plaques $(3,4)$. In the partial mind plaques, with soft texture, larger lipid necrotic core, thin fibrous cap, the collagen content and the production of collagen fibers, smooth muscle cells (SMCs) decreased significantly, including a large number of inflammatory cell (macrophages, $\mathrm{T}$ cells and mast cells) infiltration. The fibrous cap bears most of the stress, and leads to thrombosis in fat nuclear separation in the blood and plaques. The thinner fibrous cap is associated with greater surrounding pressure, and is thus easier to break $(5,6)$.

The integrity of the fibrous cap and fracture resistance mainly depends on the extracellular matrix (ECM) constantly reshaping. ECM content, thickness and strength are very important to prevent plaque rupture. ECM synthesis and degradation maintains a dynamic balance, and matrix metalloproteinase (MMP) and tissue inhibitors of metalloproteinase (TIMP) play important roles in maintaining this balance. MMP is a set of homologous enzymes dependent on $\mathrm{Ca}^{+}$and $\mathrm{Zn}^{+}$neutral protease. MMPs degrade ECM to thin fibrous caps. TIMP are polygenes encoding the protein family, with endogenous MMP serving as a specific inhibitory factor (7-9). The TIMP inhibition for MMP activity can reduce the harm to the plaque. At present, we have found four TIMPs, including TIMP-1, -2, -3 and -4 (7). TIMP1 mainly relates to atherosclerosis $(8,9)$. Transforming growth factor- $\beta$ (TGF- $\beta$ ) is a protein peptide with a variety of functions, widely in organization, TGF- $\beta 1$ had highest proportion $(>90 \%)$ and the strongest activity in cells, and is secreted in the blood vessel walls (such as SMCs, endothelial cells and macrophages) of cells. TGF- $\beta$ can promote ECM synthesis and secretion, adjust MMP content and activity, and stimulate TIMP synthesis to play a key role in keeping the balance between atheromatous plaque inflammation and fibrosis. TGF- $\beta$ is an important factor for originating protection of atherosclerosis, as well as maintain the plaque stability $(10,11)$. As a result, we assumed 'protective factor' TGF- $\beta 1$ and restraining factor TIMP1 to be imported in vulnerable plaque inflammatory cells, to stabilize ECM to achieve stable plaques.

Ultrasound-targeted microbubble destruction (UTMD) is a rapid development non-viral vector-mediated gene transfection technique utilized because of its safety and ultrasonic irradiation target tissue specificity. UTMD has been successfully applied in a variety of tissue and organ experimental research $(12,13)$. In the present study, we combined the UTMD 
and recombinant adeno-associated virus (rAAV), to achieve a high safety and transfection, and gene expression (14). In animal models, when the target therapy of TGF- $\beta 2$, combined with RNAi intervention technology, and hyperplasia of fundus change in PVR models were significantly suppressed, the treatment effect was significantly improved (15).

At present, UTMD is mainly directed towards myocardial ischemia (13). Therefore, in the present study, on the basis of previous research, using IRES sequence, we determined whether ultrasound-targeted microbubble destruction (UTMD) combined with dual targeting of transforming growth factor (TGF)- $\beta 1$ and tissue inhibitors of metalloproteinase (TIMP) 1 recombinant adeno-associated virus (rAAV) can stabilize atherosclerotic vulnerable plaques. The results showed that TGF- $\beta 1$ and TIMP1 are crucial in the regulation of atherosclerotic plaques.

\section{Materials and methods}

Animals. To establish the carotid atherosclerotic vulnerable plaque rabbit model, 18 New Zealand healthy white male rabbits, weight 2.3-2.9 kg, 4-5 months of age were provided by the Naval Medical Research Institute. Starting from week 1, experimental rabbits were given high fat (1\% cholesterol) feed for up to 10 weeks. After 2 weeks, the experimental rabbits were anesthetized with $3 \%$ pentobarbital $30 \mathrm{mg} / \mathrm{ml} / \mathrm{kg}$ after ear marginal vein anesthesia. A proximal left thigh line was inserted into the left femoral artery to separate the skin and subcutaneous tissue, and femoral of $2 \mathrm{~cm}$ was isolated. Ligation telecentric with surgical suture near the heart filled a femoral artery to block blood flow, and a ' $V$ ' type small mouth artery wall was cut. In retrograde manner, a balloon catheter (diluted with 1:15 heparin saline infiltration) was inserted to thoracic aorta (about $20 \mathrm{~cm}$ ) using thread, and a 20-ml syringe, a $10-\mathrm{ml}$ air injection was used to fill the balloon, after which the syringe was gradually pulled back until the common iliac artery (about 10-cm scale) allowing for insertion of the catheter. The syringe was pulled once again ensuring the intimal injury, prior to removing the catheter. Ligation followed and skin and subcutaneous tissue were obtained prior to washing the wound with penicillin sodium. Four weeks after confirmation of the model, the model group was injected with saline, the UTMD vector group was injected with UTMD vector and the UTMD rAAV group was injected with UTMD targeted rAAV. The injection was given once per week, for up to 10 weeks.

The animal experiments were approved by the Ethics Committee of Shanghai General Hospital Affiliated to Nanjing Medical University.

Hematoxylin and eosin $(H \& E)$ staining. The right common carotid artery was selected in the rabbits midway through embedding $1 \mathrm{~cm}$. Each group of blood vessels was placed in the same wax block (3 replicates of thickness 3-4 $\mu \mathrm{m}$ at $10 \mu \mathrm{m}$ intervals). After H\&E staining, the tissues were observed at x10 magnification.

THP-1 macrophage cell induction. THP-1 cells were purchased from ATCC. Mononuclear THP-1 cells were cultured in RPMI-1640 medium containing $10 \%$ heat-inactivated fetal bovine serum, $10 \mu / \mathrm{ml}$ penicillin and $10 \mu \mathrm{g} / \mathrm{ml}$ of streptomycin, and incubated at $37^{\circ} \mathrm{C}$ and $5 \% \mathrm{CO}_{2}$ prior to maintaining the cell number at $1 \times 10^{8} /$ flask. The subculture THP-1 cells were diluted at $1 \times 10^{6} / \mathrm{ml}$, vaccinated in $35-\mathrm{mm}$ Petri dishes containing $100 \mathrm{ng} / \mathrm{ml}$ phorbol esters (PMA), $0.3 \%$ BSA serum-free RPMI-1640 medium, and stimulated for $48 \mathrm{~h}$ to induce differentiation. We used light microscope (Olympus, Tokyo, Japan) to observe the morphology, and for the identification of differentiation to macrophages.

RT-PCR mRNA expression. Total RNA was extracted using Total RNA kit (Toyobo, Osaka, Japan). SYBR-Green RT-PCR was performed and GAPDH mRNA was used to normalize the RNA inputs. The primers were produced by Shanghai Sangon Biological (Shanghai, China). RT-PCR was used to detect the target gene expression TGF- $\beta 1$ and TIMP1. Total RNA was extracted for RNA reverse transcription cDNA.

Immunofluorescence. After transfection for $24 \mathrm{~h}$, the cells in each group washed three times and fixed in $4 \%$ paraformaldehyde for $15 \mathrm{~min}$ in PBS, permeabilized with $0.1 \%$ Triton X-100, and blocked with $1 \%$ BSA in PBS for $1 \mathrm{~h}$. Cell nuclei were dyed with DAPI (Vector Laboratories, Burlingame, CA, USA). After washing, the cells were visualized using a confocal laser microscope system (Nikon, Tokyo, Japan).

Transfection efficiency and cell apoptosis detection. Each cell group was transfected for $48 \mathrm{~h}$ and then digested with serum free medium suspension cells. According to Annexin V-FITC apoptosis kit manual introduction, flow cytometry was used to test transfection and cell apoptosis.

Western blotting. Cells were collected in a lysis buffer, the protein quantification was determined by BCA protein kit (Thermo Fisher Scientific, Waltham, MA, USA) and equal amounts of proteins were separated by $12 \%$ SDS-PAGE as described elsewhere (16). GAPDH was chosen as an internal control.

Statistical analysis. Data were presented as the mean \pm standard deviation. Statistical analysis was carried out using Prism 5.0 (Prism; GraphPad Software, Inc., La Jolla, CA, USA). One-way analysis of variance and post hoc comparisons were used for the statistical evaluation. $\mathrm{P}<0.05$ was considered to indicate a statistically significant difference.

\section{Results}

Protein expression with H\&E staining and western blotting. H\&E staining results showed that after treating UTMD with TGF- $\beta 1$ and TIMP1 rAAV, the therapy group showed significantly stabilized atherosclerosis vulnerable plaques (Fig. 1A). We also examined the protein expression level of TGF- $\beta 1$ and TIMP1 factors that have higher expression in therapy group than the model group (Fig. 1B).

Mononuclear cell morphological observation. THP-1 cells were observed under an inverted microscope had equal size small round cells, surface was smooth with adherent growth (Fig. 2A). PMA was used for $48 \mathrm{~h}$ induction to produce macrophages, and found that some cells formed circular to 
A
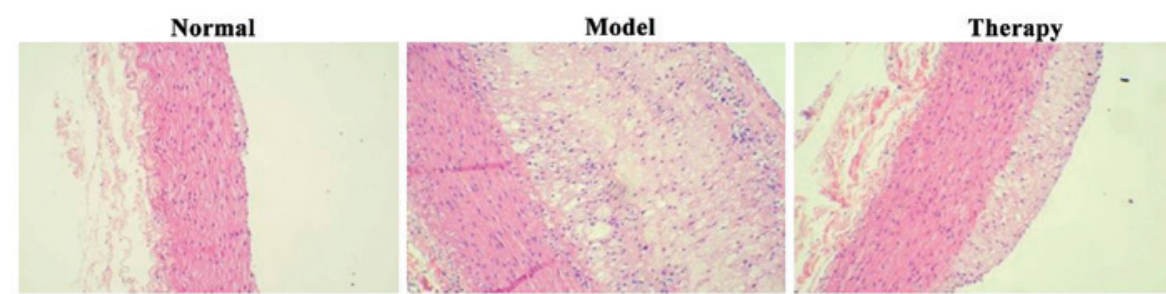

B
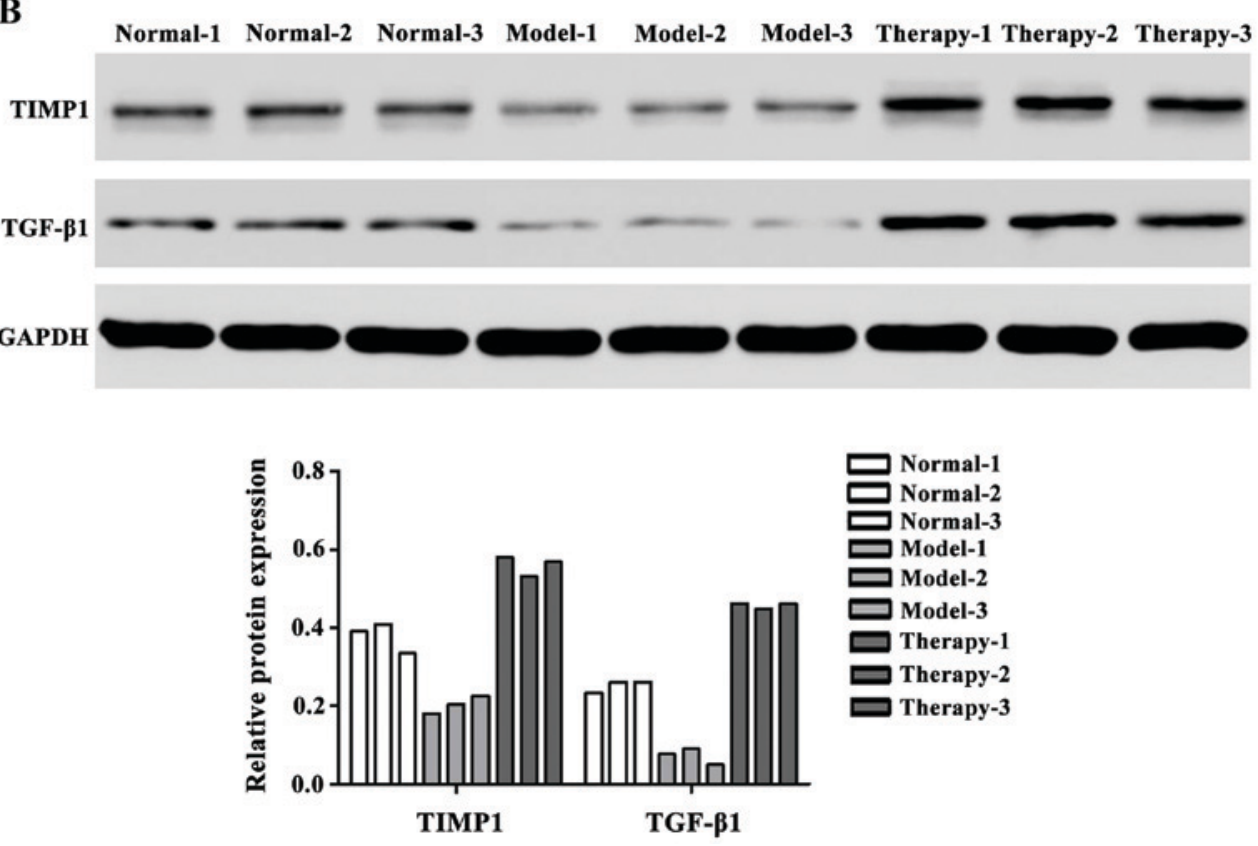

Figure 1. The therapy results in a rabbit model. (A) H\&E staining result. (B) Western blotting to TGF- $\beta 1$ and TIMP1 protein level. H\&E, hematoxylin and eosin; TGF- $\beta 1$, transforming growth factor- $\beta 1$; TIMP1, tissue inhibitors of metalloproteinase 1 .
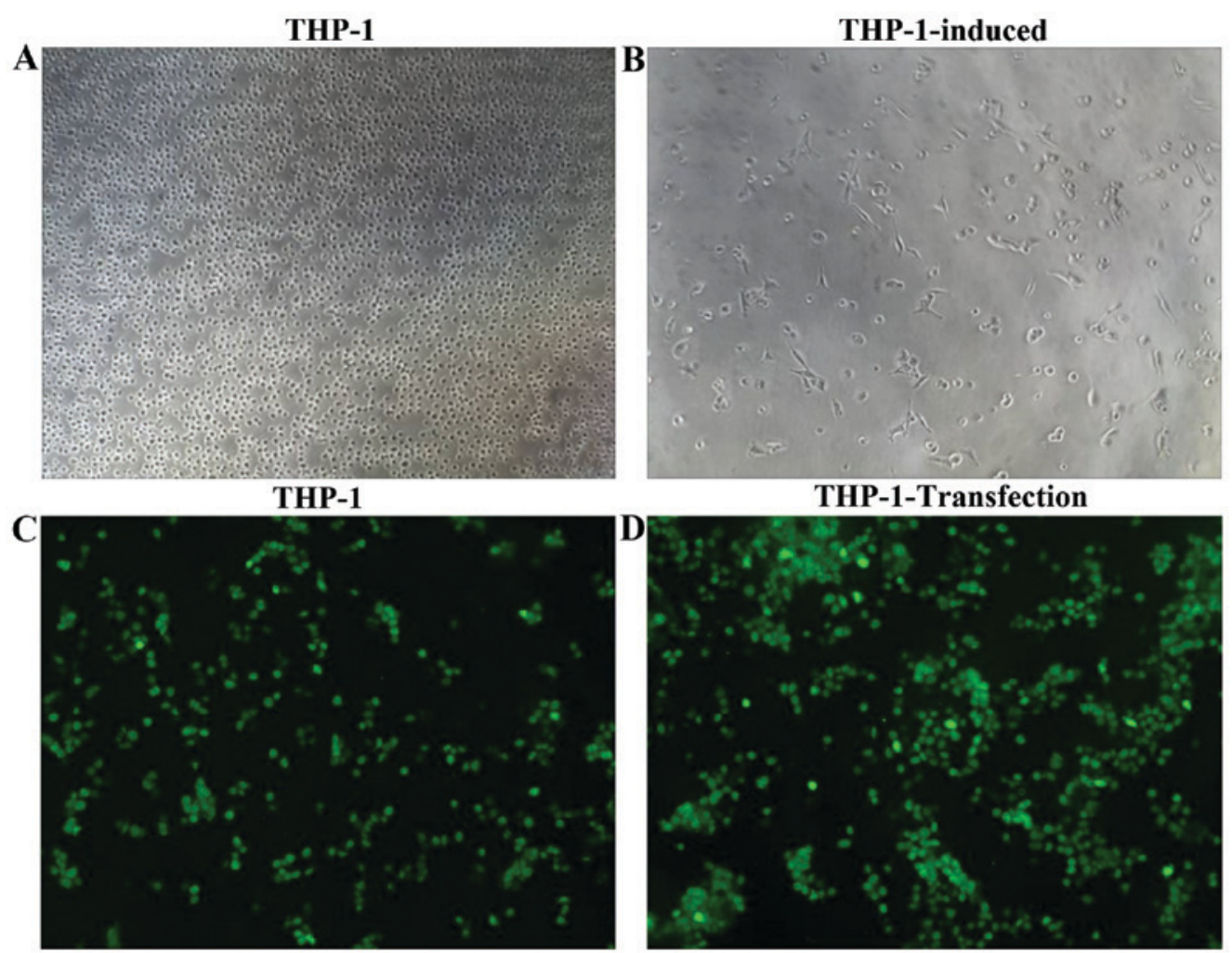

Figure 2. THP-1 cells were induced into macrophages. (A) Normal THP-1 cells. (B) Macrophages were induced by PMA after 48 h (magnification, x100). (C) Expression of THP-1 transfection detected by immunofluorescence (indicated by green fluorescence) after treatment for $24 \mathrm{~h}$. The normal control (NC).

(D) The transfection cell model. 
A

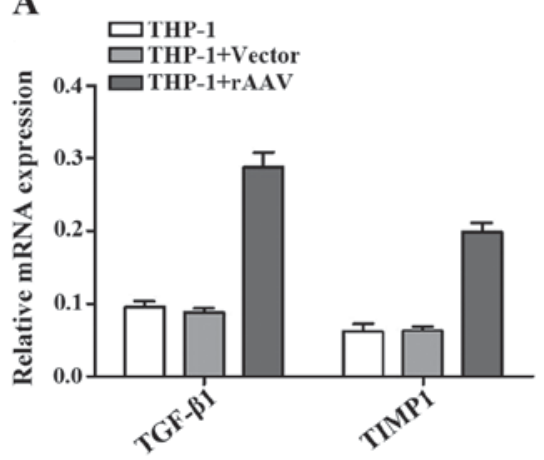

B

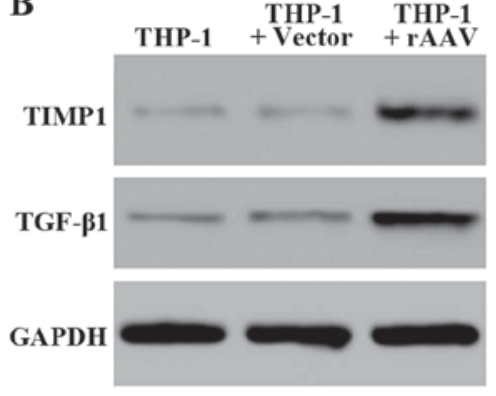

C

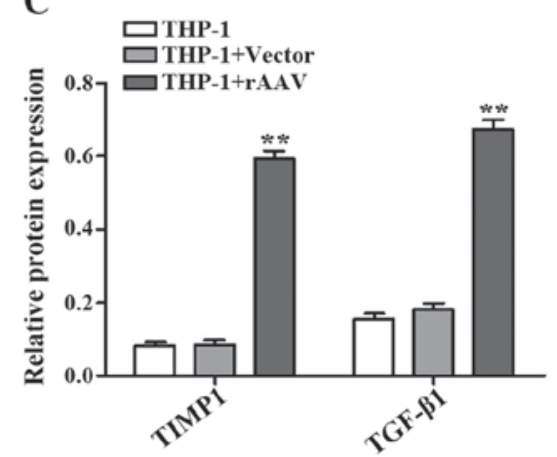

Figure 3. The mRNA and protein expression level in THP-1 macrophages. (A) The mRNA expression levels of TGF- $\beta 1$ and TIMP1 were detected. (B and C) The protein expression levels of TGF- $\beta 1$ and TIMP1 level were detected. ${ }^{* *}$ Compared with THP- $1+$ Vector group, P<0.01. TGF- $\beta 1$, transforming growth factor- $\beta 1$; TIMP1, tissue inhibitors of metalloproteinase 1.
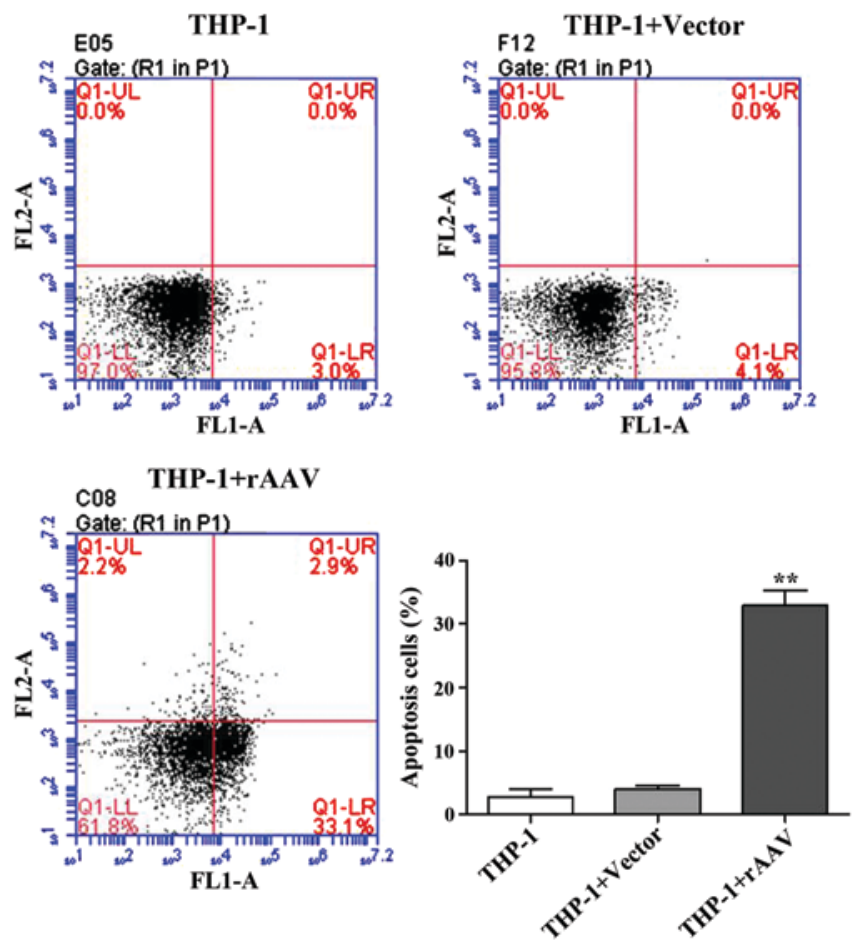

Figure 4. Cell apoptosis rates in THP-1 macrophages. Compared to the control group, the rAAV group apoptosis was significantly induced. rAAV, recombinant adeno-associated virus. ${ }^{* *} \mathrm{P}<0.05$, significant difference compared to THP-1 + Vector group.

polygon shape under the inverted microscope. Additionally, the cells showed stick walls, strong adhesion, the volumes increased and a few revealed long fusiform shapes (Fig. 2B).

Transfection THP-1 mRNA and protein expression level. After using UTMD-transfected genes, we tested the TGF- $\beta 1$ and TIMP1 mRNA and protein expression level. The GAPDH was used as an internal control to quantify mRNA expression (Fig. 3).

Apoptosis result. After UTMD treatment, flow cytometry was performed to examine the cells apoptosis. The results showed that after transfection with TGF- $\beta 1$ and TIMP1 rAAV, the apoptotic rates were significantly induced from 4.1 to $33.1 \%$ in the rAAV group (Fig. 4).

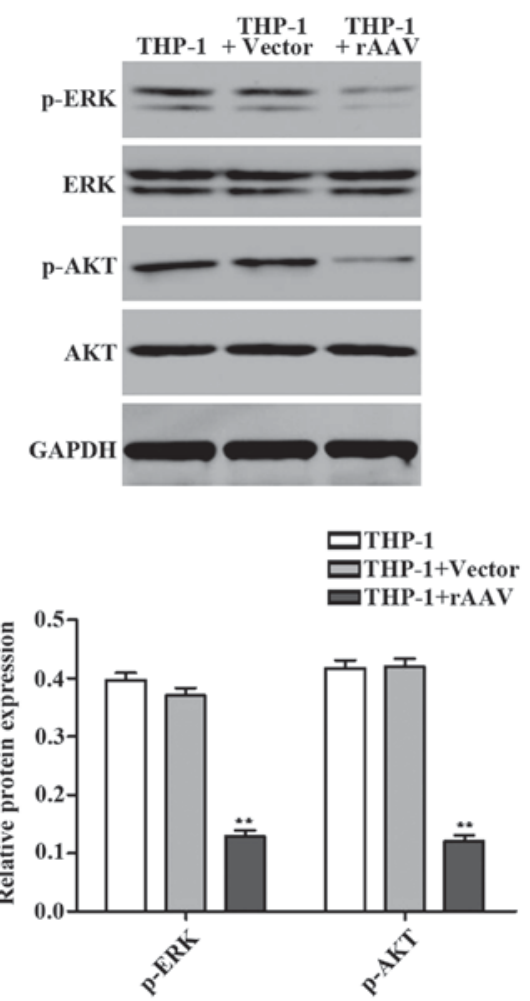

Figure 5. Western blotting for apoptosis-related target gene expression. ${ }^{* *} \mathrm{P}<0.05$, significant difference compared to THP-1 + Vector group.

Western blot results. We detected expression of apoptosisrelated proteins ERK and AKT using western blotting. It was found that the protein levels of p-ERK and p-AKT in rAAV group were significantly downregulated compared to the control group (Fig. 5). These data indicated that TGF- $\beta 1$ and TIMP1 rAAV induced cell apoptosis by regulating ERK and AKT signaling proteins.

\section{Discussion}

This study tested the hypothesis that a specific TGF- $\beta 1$ and TIMP1 influence the development of atherosclerotic plaques. rAAV have some reported biological features such as non-pathogenic nature, broad host range, ability to infect dividing and non-dividing cells and long-term expression 
of foreign genes (17). UTMD has been shown to be able to enhance adenoviral transfection efficiency (18). In this study, we used UTMD enhanced rAAV-mediated gene TGF- $\beta 1$ and TIMP transfer into models and cells.

The rabbit model provided direct evidence that the TGF- $\beta 1$ and TIMP1 protein levels were significantly upregulated in the therapy group. Furthermore, H\&E staining showed that the athrosclerosis vulnerable plaques were stabilized in the therapy group compared to the model group. TGF- $\beta 1$ is an important cytokine in the atherogenic process preventing atherosclerotic plaque formation (19). TIMP1 is synthesized by most types of connective tissue cells as well as macrophages. The overexpression of TIMP1 has been reported to reduce atherosclerotic lesion in $\mathrm{ApoE}^{-/-}$mice (20).

We also demonstrated the UTMD delivered TGF- $\beta 1$ and TIMP1 genes by rAAV into THP-1 macrophages. Apoptosis plays an essential role in different pathological processes including atherosclerosis, in which it affects all cell types in the atherosclerotic lesion (21). The apoptosis rates were significantly induced after UTMD treatment compared to the control group. ERK is involved in cell death (22). AKT plays a role in the development of resistance to apoptosis (23). We showed that rAAV group has downregulated p-ERK and p-AKT apoptosis-related protein levels compared to the control group. These studies provided evidence for the important roles of TGF- $\beta 1$ and TIMP1 in athrosclerosis plaques.

As TGF- $\beta 1$ and TIMP1 have been demonstrated to be involved in atherosclerotic plaques, we concluded that TGF- $\beta 1$ and TIMP1 may therefore beneficially modulate the plaque stability.

\section{References}

1. Anogeianaki A, Angelucci D, Cianchetti E, D'Alessandro M, Maccauro G, Saggini A, Salini V, Caraffa A, Tete S, Conti F, et al: Atherosclerosis: a classic inflammatory disease. Int J Immunopathol Pharmacol 24: 817-825, 2011.

2. Hansson GK: Atherosclerosis - an immune disease: the Anitschkov Lecture 2007. Atherosclerosis 202: 2-10, 2009.

3. Muller JE, Abela GS, Nesto RW and Tofler GH: Triggers, acute risk factors and vulnerable plaques: the lexicon of a new frontier. J Am Coll Cardiol 23: 809-813, 1994.

4. Fishbein MC: The vulnerable and unstable atherosclerotic plaque. Cardiovasc Pathol 19: 6-11, 2010.

5. Virmani R, Burke AP, Farb A and Kolodgie FD: Pathology of the unstable plaque. Prog Cardiovasc Dis 44: 349-356, 2002.

6. Bartels ED, Bang CA and Nielsen LB: Early atherosclerosis and vascular inflammation in mice with diet-induced type 2 diabetes Eur J Clin Invest 39: 190-199, 2009.

7. Massova I, Kotra LP, Fridman R and Mobashery S: Matrix metalloproteinases: structures, evolution, and diversification. FASEB J 12: 1075-1095, 1998.

8. Orbe J, Fernandez L, Rodríguez JA, Rábago G, Belzunce M, Monasterio A, Roncal C and Páramo JA: Different expression of MMPs/TIMP-1 in human atherosclerotic lesions. Relation to plaque features and vascular bed. Atherosclerosis 170 269-276, 2003
9. Fiotti N, Altamura N, Orlando C, Simi L, Reimers B, Pascotto P, Zingone B, Pascotto A, Serio M, Guarnieri G, et al: Metalloproteinases-2, -9 and TIMP-1 expression in stable and unstable coronary plaques undergoing PCI. Int J Cardiol 127: 350-357, 2008

10. Bot PT, Grundmann S, Goumans M-J, de Kleijn D, Moll F, de Boer O, van der Wal AC, van Soest A, de Vries JP, van Royen N, et al: Forkhead box protein P1 as a downstream target of transforming growth factor- $\beta$ induces collagen synthesis and correlates with a more stable plaque phenotype. Atherosclerosis 218: 33-43, 2011.

11. Deng H-B, Jiang CQ, Tomlinson B, Liu B, Lin JM, Wong KS, Cheung BM, Lam TH and Thomas GN: A polymorphism in transforming growth factor- $\beta 1$ is associated with carotid plaques and increased carotid intima-media thickness in older Chinese men: the Guangzhou Biobank Cohort Study-Cardiovascular Disease Subcohort. Atherosclerosis 214: 391-396, 2011.

12. Walton CB, Anderson CD, Boulay R and Shohet RV: Introduction to the ultrasound targeted microbubble destruction technique. J Vis Exp 12: 2963, 2011.

13. Fujii H, Li S-H, Wu J, Miyagi Y, Yau TM, Rakowski H, Egashira K, Guo J, Weisel RD and Li R-K: Repeated and targeted transfer of angiogenic plasmids into the infarcted rat heart via ultrasound targeted microbubble destruction enhances cardiac repair. Eur Heart J 32: 2075-2084, 2011

14. Li HL, Zheng XZ, Wang HP, Li F, Wu Y and Du LF: Ultrasound-targeted microbubble destruction enhances $\mathrm{AAV}$-mediated gene transfection in human RPE cells in vitro and rat retina in vivo. Gene Ther 16: 1146-1153, 2009.

15. Zheng X, Du L, Wang H and Gu Q: A novel approach to attenuate proliferative vitreoretinopathy using ultrasound-targeted microbubble destruction and recombinant adeno-associated virus-mediated RNA interference targeting transforming growth factor- $\beta 2$ and platelet-derived growth factor-B. J Gene Med 14: 339-347, 2012.

16. Padda RS, Gkouvatsos K, Guido M, Mui J, Vali H and Pantopoulos K: A high-fat diet modulates iron metabolism but does not promote liver fibrosis in hemochromatotic $\mathrm{Hjv}^{-/}$mice. Am J Physiol Gastrointest Liver Physiol 308: G251-G261, 2015.

17. Daya $S$ and Berns KI: Gene therapy using adeno-associated virus vectors. Clin Microbiol Rev 21: 583-593, 2008.

18. Howard CM, Forsberg F, Minimo C, Liu JB, Merton DA and Claudio PP: Ultrasound guided site specific gene delivery system using adenoviral vectors and commercial ultrasound contrast agents. J Cell Physiol 209: 413-421, 2006.

19. Mallat Z, Gojova A, Marchiol-Fournigault C, Esposito B, Kamaté C, Merval R, Fradelizi D and Tedgui A: Inhibition of transforming growth factor- $\beta$ signaling accelerates atherosclerosis and induces an unstable plaque phenotype in mice. Circ Res 89: 930-934, 2001.

20. Rouis M, Adamy C, Duverger N, Lesnik P, Horellou P, Moreau M, Emmanuel F, Caillaud JM, Laplaud PM, Dachet C, et al: Adenovirus-mediated overexpression of tissue inhibitor of metalloproteinase-1 reduces atherosclerotic lesions in apolipoprotein E-deficient mice. Circulation 100: 533-540, 1999.

21. Guevara NV, Chen K-H and Chan L: Apoptosis in atherosclerosis: pathological and pharmacological implications. Pharmacol Res 44: 59-71, 2001.

22. Cagnol S and Chambard JC: ERK and cell death: Mechanisms of ERK-induced cell death - apoptosis, autophagy and senescence. FEBS J 277: 2-21, 2010

23. Khwaja A: Akt is more than just a Bad kinase. Nature 401: 33-34, 1999. 